\title{
Major memory for microblogs
}

\author{
Laura Mickes • Ryan S. Darby • Vivian Hwe • \\ Daniel Bajic • Jill A. Warker • Christine R. Harris • \\ Nicholas J. S. Christenfeld
}

Published online: 12 January 2013

(C) Psychonomic Society, Inc. 2013
Keywords Memory $\cdot$ Microblog $\cdot$ Facebook . Levels of processing

The online world permits its citizens to post their every thought as blogs, Facebook status updates, forum comments, and the like. Not every such posting is extraordinarily interesting; indeed, Facebook posts have been described as "navelgazing ... spam" (Griggs, 2009). However mundane, or even inane, such sharing may be, it is also enormously popular. On Facebook alone, people collectively post over thirty million times an hour (Facebook.com, 2011). This popularity suggests that something about such "microblogging" resonates with human nature. We explored this question in a series of experiments examining the memorability of microblog posts. If microblogs are as vacuous as some propose, they should vanish quickly from one's mind. On the other hand, as such outputs represent the natural, essentially unfiltered, emanations of human minds, perhaps they also have a special place there as input. The success of Facebook, after all, depends not just on people's willingness to post their thoughts, but also on their willingness to read those posts.

Particular sorts of information may have a privileged place in memory. Nairne and Pandeirada (2010) suggested that our memory systems developed to solve adaptive problems and that we should see enhanced memory in tasks that tap into the types of issues confronted in our ancestral past. Nairne and colleagues have shown, for example, better memory for information that would have been related to survival needs (Nairne, Pandeirada, \& Thompson, 2008; Nairne, Thompson, \& Pandeirada, 2007; see also Kang, McDermott, \& Cohen, 2008), and social information could likewise be privileged because of our ancestral reliance on group living. Indeed, recently Klein, Cosmides, Gangi, Jackson, and Tooby (2009) described memory as "an adaptive tool for sociality" (p. 284). The research on social memory thus far has focused on autobiographical memory 
(e.g., birthdays; Merz, Wolf, \& Hennig, 2010) or face-name pairs (e.g., Takahashi et al., 2004). The popularity of microblogging affords a way to investigate memory for the daily details of social information.

Baumeister and Leary (1995) proposed that social belongingness is so essential in humans that it should be considered a basic, innate motive. Humans are driven to share their experiences with others-an inclination that emerges early in development, with preverbal infants' use of pointing and eye gaze in social interactions - and this feature seems to separate us from even our ape cousins. This need to express and share our experiences has been variously documented (see Emery, 2000). Such views support the popularity of Facebook posts and other microblogs and suggest that, if receiving these communications is as much a part of our nature as making them, these reports could be particularly memorable.

\section{Experiment 1}

In Experiment 1, we examined the strength of memory for Facebook status updates (i.e., messages posted to friends). We compared the memorability of such microblogs to that for sentences from published books (Exp. 1a) and to the memorability of human faces (Exp. 1b).

\section{Experiment 1a}

\section{Method}

Participants Thirty-two University of California, San Diego (UCSD) undergraduates (age: $M=21.00$ years, $S D=2.14$; 27 female, five male) were randomly assigned to one of two conditions, with half in the Facebook condition and half in the book condition.

Materials Five undergraduate research assistants, who were blind to the hypothesis, gathered 200 posts written by others (from, collectively, over 3,000 friends) from their own Facebook feeds. Each post was the most current for its author, and none was connected with games, links, or photos (see the Appendix for examples).

A total of 200 sentences were also selected from books on amazon.com, using the "Last 30 days" option under the "New Releases" section, including both fiction and nonfiction. For each of these books for which the "Look Inside" feature was available, we used the "Surprise Me!" option, and within that random page, a single sentence was randomly selected. Sentences with quotations, single-word sentences, and sentences that contained more than 25 words were not selected (see the Appendix for examples).
Of the 200 stimuli in each condition, 100 were randomly selected as targets for each participant to memorize during the study phase. The other 100 were reserved as lures for the testing phase, to assess whether the participants could identify stimuli they had seen before: (a different combination of targets and lures per participant). Instructions and stimuli were displayed via E-Prime (Psychology Software Tools Inc., Sharpsburg, PA; www.pstnet.com) on a 22 -in. monitor in 18-point Courier font.

Procedure The participants were informed from the outset that they were participating in a memory experiment. After a practice trial, the study phase began. During study, 100 targets were individually presented for 3,000 ms, followed by a blank-screen interstimulus interval (ISI) of $250 \mathrm{~ms}$. Immediately after the study phase, participants took a selfpaced recognition test that consisted of the 100 targets randomly intermixed with 100 lures. Participants indicated their confidence that each post had been previously seen (old) or not previously seen (new) on the study list, using a 20-point rating scale that is shown in Fig. 1 (Mickes, Wixted, \& Wais, 2007). A keypress of " 1 " indicated that they were $100 \%$ certain that the post had not appeared in the study list, and a keypress of " 20 " represented that they were $100 \%$ confident that an item had appeared. Thus, a correct response for a lure would be between 1 and 10 , and a correct response for a target would be between 11 and 20 .

\section{Results}

Participants in the Facebook condition responded generally with the highest levels of confidence and were highly accurate. The average $d^{\prime}$ (an unbiased measure of memory strength) in the Facebook condition $(M=2.48, S D=$ $0.69)$ was significantly greater than the average $d^{\prime}$ in the book sentence condition $(M=1.72, S D=0.54), t(30)=$ $3.47, p=.002$. The percent correct measure (calculated as the number of hits plus correct rejections, divided by 200 trials), while potentially biased (Macmillan \& Creelman, 2005), is intuitively appealing, and it reveals exactly the same story: significantly greater memory for Facebook posts $(M=85 \%, S D=7.2)$ than for sentences from books $(M=$ $76 \%, S D=6.2), t(30)=3.72, p=.001$.

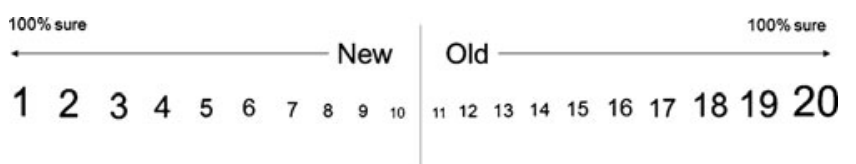

Fig. 1 Confidence rating scale used in the experiments. A response of 1 indicated that the participant was $100 \%$ certain that an item had not appeared during the study phase, and a response of 20 indicated that he or she was $100 \%$ certain that an item had appeared. The different font sizes visually represent the levels of confidence. This scale was presented for each test item in the experiments 
To generate the receiver-operating characteristic (ROC) plot, hit and false alarm rate pairs were computed for each level of confidence. For example, in the Facebook condition, $56 \%$ of the targets and $6 \%$ of the lures received a confidence rating of 20 (i.e., a hit rate of $56 \%$ and false alarm rate of $6 \%$ for ratings of 20). Next, another hit and false alarm rate pair was obtained by computing the percentages of targets and lures that received ratings of 19 and 20. The ratings were cumulated in this manner until we had generated 19 separate hit and false alarm rate pairs, which are plotted in the ROC. The farther the operating points that make up the ROC curve are from the diagonal, or "chance" line (i.e., the closer they are to the upper left corner), the greater the discriminability between targets and lures (Macmillan \& Creelman, 2005). Figure 2 displays the ROC data, and it clearly illustrates that individuals in the Facebook condition discriminated targets from lures much more easily than did those in the book sentence condition.

Before investigating deeper conceptual explanations for the advantage that Facebook posts seem to enjoy, we ruled out various more superficial possibilities. First, we tested whether the length of the post or sentence was a critical factor. The Facebook posts $(M=11.49, S D=6.54)$ had significantly, albeit slightly, more words than the book sentences $(M=10.27, S D=4.00), t(199)=6.14, p<.001$. To adjust for this, we did a median split on the numbers of words for the Facebook posts and book sentences (median = 10.00 for both), and then compared the $d^{\prime}$ scores for the shorter Facebook posts and the longer sentences. The average $d^{\prime}$ for the Facebook posts $(M=2.67, S D=0.98)$, was still significantly higher than that for the book sentences $(M=1.77$,

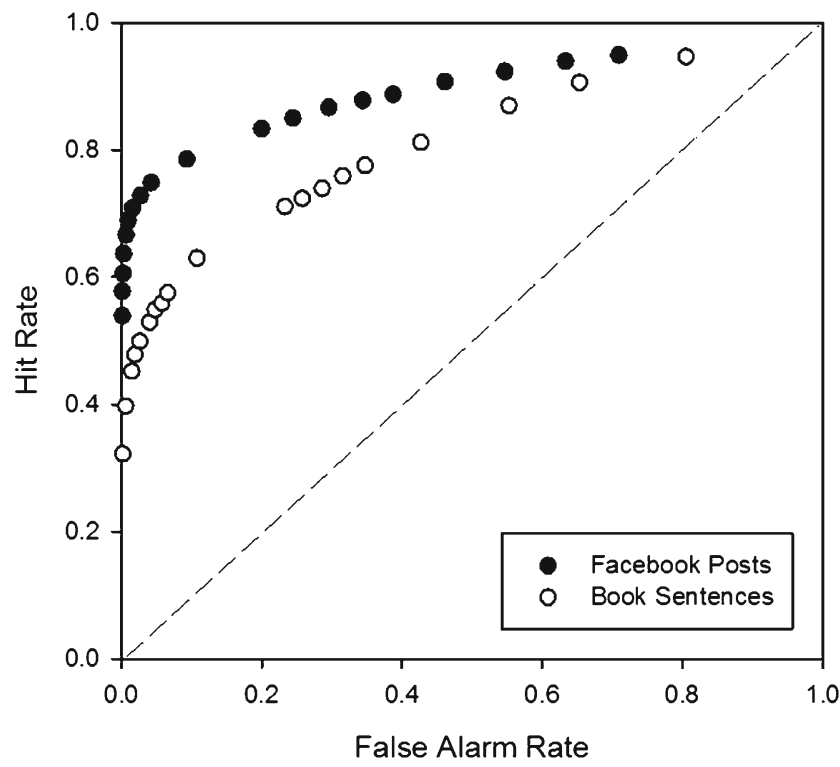

Fig. 2 Group receiver-operating characteristic (ROC) data for the Facebook posts and book sentences from Experiment 1a. The dashed line represents chance performance
$S D=0.92), t(102)=6.86, p<.001$, even with the length confound reversed, suggesting that the Facebook posts' advantage was not due to length. ${ }^{1}$

Another possibility is that Facebook posts may capitalize on perceptual matching of surface-level differences (e.g., Mandler, 1979), since they are littered with irregular typography. To test this, we separated posts that contained emoticons, multiple exclamation points, all letters capitalized, or multiple, repeated letters (e.g., "Del Mar Opening Day is on my birthday this year!!! :) Hellooo HATS") from posts that did not. A total of 86 posts did not include any of these components. Memory was still significantly higher for these orthographically regular posts $\left(d^{\prime}: M=2.57, S D=1.18\right)$ than for the book sentences $\left(d^{\prime}: M=1.95, S D=1.07\right)$, $t(284)=4.32, p<.001$.

\section{Discussion}

Memory was substantially higher for Facebook posts than for book sentences. Explanations for this advantage that were based merely on surface-level differences were ruled out: The difference was not due to posts containing emoticons, unique characters, or many or few words; the advantage persisted when all such posts were removed. The posts culled from Facebook showed remarkable memorability; memory for book sentences, on the other hand, reflected more typical memory performance. For example, the memory for sentences found here seems similar to results reported by Belmore (1982) using similar recognition tests for sentences with roughly the same number of targets and lures as in our experiment.

Clearly participants recognized Facebook posts better than ordinary published sentences. To get a further sense of the memorability of such posts, we next compared them to memory for faces. A region of the brain, the fusiform face area, is dedicated to face processing (e.g., Kanwisher, McDermott, \& Chun, 1997), suggesting that the brain is specially designed to process and store facial information. While many factors can influence the memorability of a set of faces, faces nonetheless can provide some calibration for the magnitude of Facebook's memorability, measuring whether memory for Facebook posts is particularly strong or memory for sentences from books is particularly weak. In Experiment $1 \mathrm{~b}$, accordingly, participants completed a memory task for faces and for Facebook posts.

\footnotetext{
${ }^{1}$ The difference in $d$ 's between short $(M=2.35, S D=1.41)$ and long $(M=2.67, S D=0.98)$ Facebook posts was marginally significant, $t$ $(198)=1.83, p=.057$. The same analysis yielded opposite results for the book sentences: Memory was significantly better, as measured by $d^{\prime}$, for short book sentences $(M=2.15, S D=1.18)$ than for long book sentences $(M=1.77, S D=0.92), t(198)=2.60, p=.010$. Neither effect, of course, can account for the superior memory for Facebook posts relative to book sentences.
} 
Experiment 1b

\section{Method}

Participants Sixteen UCSD undergraduates (age: $M=$ 20.25 years, $S D=1.34 ; 11$ female, five male) participated for course credit.

Materials Four undergraduate assistants used their own Facebook accounts to find a new sample of 200 posts by others $^{2}$ (see the Appendix). Using a new set of Facebook posts allowed an independent replication of their memorability. For the face memory task, 200 neutral faces (frontal view only) were selected from the Color FERET database (http://face.nist.gov/colorferet/), with 100 of each stimulus type randomly chosen to be targets and 100 to be lures (a different combination of targets and lures per participant).

Procedure The procedure was identical to that of Experiment 1a, with two changes: Faces were used instead of book sentences, and a within-subjects rather than between-subjects design was used. Task order was counterbalanced; half of the participants completed the face memory task first, and half completed the Facebook memory task first. Again, the presentation time for each target was 3,000 ms, with a 250-ms ISI. Immediately after the study phase, participants took a self-paced recognition test that consisted of 100 targets randomly intermixed with 100 lures, shown one at a time, and the participants provided $\mathrm{old} /$ new responses on a 20 -point rating scale.

\section{Results}

We found no order effects, so the data from the two orders were combined for analyses. Accuracy was much higher for Facebook posts than for faces $\left(d^{\prime}: M=2.51, S D=0.37\right.$, vs. $M=0.95, S D=0.67$, respectively), $t(15)=11.70$, $p<.001$. Figure 3 shows the ROC data, and as in Experiment 1a, participants easily discriminated targets from lures when the stimuli were Facebook posts. Facebook posts are particularly memorable as compared to faces.

\section{Discussion}

Experiment $1 \mathrm{~b}$ further strengthened the idea that memory for these microblog posts is remarkable, with Facebook beating both faces and books. As a frame of reference,

\footnotetext{
${ }^{2}$ The Facebook posts (from Exp. 1b) were chosen to have a range of activities, emotions, and writing styles. The additional selection criteria made no difference to the memorability, which almost exactly matched that observed in Experiment 1a.
}

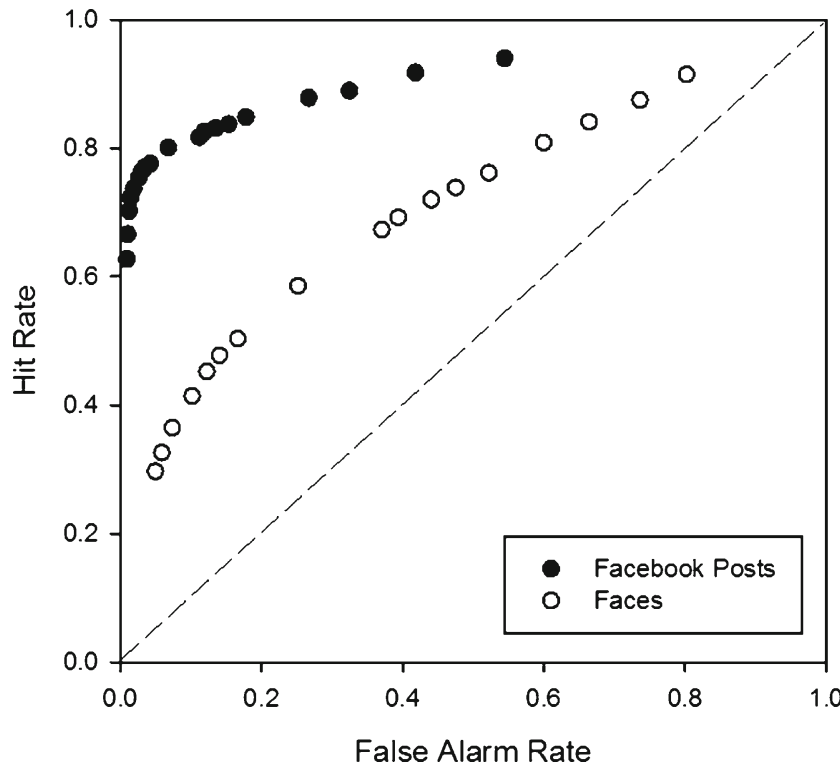

Fig. 3 Group receiver-operating characteristic (ROC) data for the Facebook posts and faces from Experiment 1b. The dashed line represents chance performance

across multiple memory tests for lists of words (Manns, Hopkins, Reed, Kitchener, \& Squire, 2003), hippocampally damaged amnesic individuals showed an average $d^{\prime}$ of 0.4 , and controls 1.5, a difference of 1.1. Averaging across our experiments, books and faces had a $d^{\prime}$ of 1.3, and Facebook a $d^{\prime}$ of 2.5, a difference of 1.2. Thus, Facebook's advantage over books and faces is on the same scale as the advantage of controls over amnesics.

While several possible mechanisms for the memory strength of Facebook posts have been ruled out, a number of explanations remain. The posts may naturally elicit social thinking and lead to stronger encoding of the posts, whereas sentences written by professional authors and unknown neutral faces may be less likely to naturally elicit such encoding-enhancing elaboration. The more gossipy nature of the Facebook posts may have been inherently more interesting to participants and contributed to this social elaboration (Schiefele \& Krapp, 1996). In addition, the Facebook posts may be particularly memorable because they are complete in and of themselves, whereas the book sentences, chosen randomly and out of context, need not have been so.

Another possible explanation is that the relatively unfiltered and spontaneous production of one person's mind is just the sort of thing that is readily stored in another's mind. The formality and complexity of well-considered and edited language, while it may have many advantages in accuracy, efficiency, and even beauty, may not be more readily stored. That is, information that people generate easily and naturally may be information that is easily and naturally remembered. Such an explanation is not entirely distinct from the 
gossip notion, since reports of the personal doings of other people may be closer to our natural speech than are more arcane and abstract reports. Two follow-up experiments explored these explanations for the superiority of Facebook posts. In the first, we examined whether spontaneous social elaboration is the secret to Facebook's success, at least in the memorability of its posts, by testing whether directed social elaboration impacts memory less for Facebook posts than for other sentences. The second was designed to compare news sentences to news headlines and to readers' comments to explore the roles of content and completeness in memorability.

\section{Experiment 2}

Facebook posts may naturally elicit social thinking (e.g., "that is something my friend Emily would post"), while published sentences from books may be much less likely to elicit such elaboration. We tested whether such elaboration underlies the Facebook effect by manipulating the level of processing in an incidental learning task (e.g., Craik \& Lockhart, 1972; Nairne et al., 2008). A shallow-encoding condition required participants to count the words of each sentence or post. A deep-encoding condition required participants to think of someone whom they knew (personally or a fictional character) who could have composed each post or sentence. If people spontaneously elaborate Facebook posts but not book sentences, the directions to elaborate should have little memory-enhancing effect on the former and a more profound effect on the latter. If, on the other hand, the Facebook advantage is not due to social elaboration, then adding it to each should have essentially equal effects.

\section{Method}

Participants Sixty-four UCSD undergraduates participated for course credit (age: $M=20.34$ years, $S D=2.12$; 41 female, 23 male). The participants were randomly assigned to Facebook posts or book sentences, as well as to either shallow or deep encoding.

Materials The Facebook posts and book sentences were nearly the same as in Experiment 1a, but to control for length, we used a subset of the book sentences and added more, chosen in the same way (to return the total number to 200 sentences), so that the Facebook posts $(M=11.64, S D$ $=6.77$ ) would not have more words than the book sentences $(M=11.21, S D=4.93), t(398)=0.72, p=.47$.

Procedure The experiment employed an incidental-learning task with a $2 \times 2$ design (shallow vs. deep processing, and book sentences vs. Facebook posts). Participants in the shallow-encoding condition counted and entered the number of words per Facebook post or book sentence. In the deepencoding condition, participants rated (on a 5-point scale, from difficult to easy) the ease with which a Facebook post or book sentence reminded them of something that someone they know would write or say (e.g., themselves, a friend, a family member, or a character in a movie or book). The instructions were adapted from Nairne et al. (2008). After a 10-min distractor task (a game of Space Invaders), the participants took a surprise recognition memory test on the 100 targets randomly intermixed with 100 lures.

Results

In the deep-encoding groups, the average ratings of the ease with which participants could think of a person who would write a sentence $(M=3.30, S D=0.42)$ or post $(M=3.20, S D=0.64)$ were not significantly different, $t(15)=0.54, p=.595$. In the shallowencoding groups, the numbers of words counted were not significantly different for book sentences $(M=$ $11.24, S D=0.42)$ and Facebook posts $(M=11.52$, $S D=0.75) ; t(30)=1.33, p=.194$

Figure 4 shows the average $d$ 's by stimulus types. A $2 \times 2$ ANOVA revealed a main effect of book sentences versus Facebook posts, with, as before, Facebook posts being remembered much better, $F(1,60)=32.90, p<.001$. There was also the predicted, and large, main effect of encoding type, with deep encoding resulting in better memorability, $F$ $(1,60)=189.26, p<.001$. There was no interaction between stimulus type and encoding type, $F(1,60)=0.75, p=.390$, suggesting that adding the encoding instructions helped the Facebook posts just as much as it helped the book sentences.



Fig. 4 Memory strength for Facebook posts and book sentences when participants were instructed to do shallow or deep processing during encoding in Experiment 2. The error bars represent standard errors of the means 


\section{Discussion}

If social elaboration were responsible for the memorability of Facebook posts, one would expect that using socially based deep encoding would equalize the $d$ 's for book sentences and Facebook posts. However, the memory benefits of Facebook posts and of social encoding were additive, suggesting that the memorability of Facebook posts in our experiment was not simply due to their engendering natural social elaboration during encoding. Furthermore, the ratings of ease of social elaboration did not suggest that the Facebook posts enjoyed any obvious natural advantage there. In the next experiment, we explored whether an item's content or completeness of ideas might account for the Facebook effect.

\section{Experiment 3}

To test the hypothesis that Facebook posts might be advantaged because they are coherent and complete ideas, we compared the memorability of sentences drawn from CNN articles to the memorability of the headlines of those articles, which are written to stand alone. To test whether the Facebook advantage might be due to their gossipy nature (e.g., general sharing of details about aspects of others' lives; Dunbar, 2004), we selected articles drawn either from news or from entertainment. Finally, to test the notion that the unfiltered, largely unconsidered postings of strangers are what are especially memorable, we also compared reader comments drawn from the comment sections at the end of each news or entertainment article to both the headlines and article sentences.

\section{Method}

Participants One-hundred-eighty UCSD undergraduates participated in exchange for course credit (age: $M=$ 20.12 years, $S D=2.50 ; 138$ female, 42 male).

Materials The stimuli consisted of text drawn from two categories: CNN's Breaking News and Entertainment News sections. From each of those categories, we drew three subcategories of stimuli: headlines, sentences, and comments. All were gathered from CNN Twitter feeds (http://twitter.com/CNNBrk and http://twitter.com/ CNNshowbiz, respectively) dating from August 12, 2011, through September 15, 2011. For each story, the tweets linked to the online article, from which we copied the headline, a sentence chosen at random from the body of the article, and a randomly chosen reader comment (in the comments section at the bottom of each article). The excluded stimuli were single-word items, items more than 25 words long, articles that contained no reader comments, and comments on comments. We gathered 200 stimuli of each of the six types. The stimuli were presented, and responses recorded, with the E-Prime software.

Procedure The participants were instructed that they would be memorizing stimuli gathered from many sources and would be given a memory test afterward. One hundred stimuli were chosen as targets, with 16 or 17 of each type randomly selected for each participant. No participant saw more than one item (headline, sentence, or comment) from a given article. One hundred lures were chosen in the same way. All other procedures were the same as in Experiment 1. Due to an experimental error, some duplicate stimuli were included. All duplicates were removed prior to analysis, resulting in the loss of two targets or lures for each of 43 subjects, and four for each of six subjects.

Results

A repeated measures ANOVA revealed significant main effects of text type (headline vs. sentence from article vs. comment) and category (breaking news vs. entertainment news) on $d^{\prime}, F$ $(2,358)=164.27, p<.001$, and $F(1,179)=320.36, p<.001$, respectively (as is shown in Fig. 5). The interaction between type of text and type of category was significant, $F(2,358)=$ $14.94, p<.001$. Testing the specific hypothesis that complete thoughts might be remembered better, we did find that, overall, the $d^{\prime}$ for headlines $(M=2.02, S D=0.82)$ was higher than that for random sentences from the articles $(M=1.59, S D=0.75)$, $p<.001$ (with Bonferroni corrections). Consistent with the idea that gossipy reports might be more memorable than news stories of a more impersonal nature, the random sentences drawn from entertainment news $(M=1.85, S D=0.72)$ were more memorable than those drawn from breaking news articles $(M=1.32, S D=0.68), p<.001$, and this held for the other

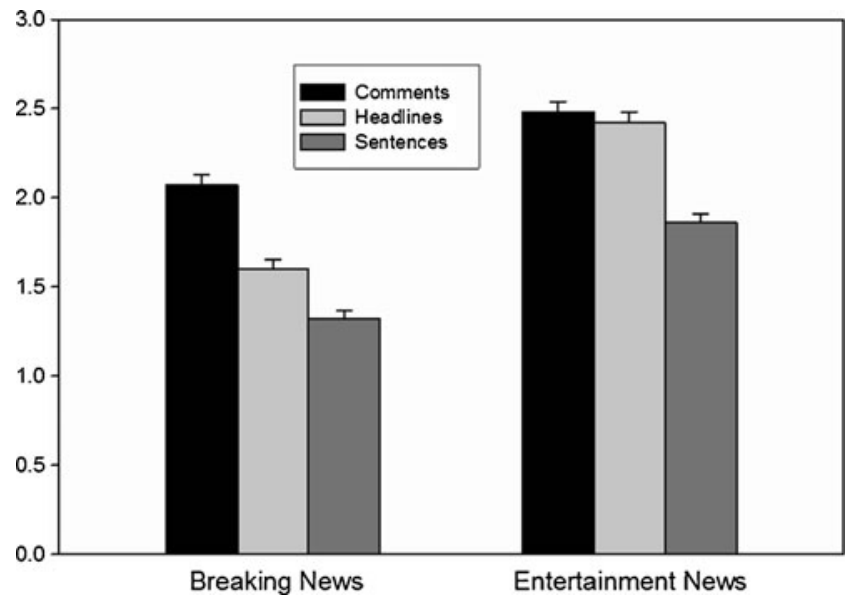

Fig. 5 Average $d^{\prime}$ values by category for Experiment 3. The error bars represent standard errors of the means 
two types of text - headlines and comments - as well. Finally, overall, comments at the ends of the articles $(M=2.27, S D=$ $0.79)$ were not only more memorable than random sentences from within the articles $(M=1.59, S D=0.75), p<.001$, but also more memorable than the headlines of those articles $(M=$ 2.02, $S D=0.82), p<.001$.

We also conducted analyses to determine whether sentence length could account for the memorability of the comments and entertainment news. A 2 (category) $\times 3$ (text type) betweengroup ANOVA was performed on the word counts for all stimuli. The interaction was not significant, $F(2,1182)=0.57$, $p>.05$. However, we did find a significant main effect for category, with higher word counts for breaking news $(M=$ $11.97, S D=5.07)$ than for entertainment news $(M=11.06$, $S D=4.84), F(1,1182)=13.14, p<.001$, as well as a significant main effect for text type (headline, $M=7.79, S D=1.93$, vs. sentence, $M=14.51, S D=4.48$, vs. comment, $M=$ $12.17, S D=5.21), F(2,1182)=270.26, p<.001$, with headlines having the lowest word count scores overall, and sentences from articles having the highest. In other words, word length is unlikely to be an explanation for the memorability differences, because the best-remembered stimuli were intermediate in length.

One might think that the greater memorability of the less formal writing might result from the use of words that are more commonly encountered in daily life. The memory literature, however, suggests that low-frequency words (e.g., acrobat) are remembered better than high-frequency words (e.g., house) on recognition memory tests (e.g., Glanzer \& Bowles, 1976). Nonetheless, we examined whether word frequency differences might account for the differences in memorability. Using the Corpus of Contemporary American English (http://corpus. byu.edu/coca/), we compared the rates of low-frequency words in headlines, comments, and sentences. Low-frequency words were here defined as those not falling within the top 3,000 words in the corpus. A 2 (category) $\times 3$ (text type) betweengroup ANOVA was performed on the percentages of words categorized as low-frequency for all stimuli. The means are shown in Table 1. A main effect of category emerged, with entertainment news using more low-frequency words than did breaking news, $F(1,1182)=21.53, p<.001$. We also found a main effect of text type, $F(2,1182)=174.91, p<.001$, with headlines having the highest number of low-frequency words. The Category $\times$ Text Type interaction was not significant, $F(2$, $1182)=1.60, p=.202$. While we did find word frequency differences, they cannot account for the observed advantage of the comments, which actually, as one might expect, had relatively few memorable low-frequency words, being very similar on that dimension to the poorly remembered sentences.

\section{Discussion}

The comments, similar to the Facebook posts in the earlier experiments, were remembered exceptionally well, especially relative to the breaking news headlines and sentences from the breaking news and entertainment articles. Their completeness of ideas (as also found in headlines) could contribute to, but not fully explain, the memorability of microblogs. Likewise, the more gossipy nature of Facebook posts (as also captured in entertainment news) could contribute to, but also not fully account for, the memorability of microblogs. It seems likely that both of these characteristics partially contribute to the memorability of microblogs. That is, the natural emanations of the human mind (as expressed in microblogs) are gossipy and have an element of completeness to them.

\section{General discussion}

In this article, we introduced and investigated a new phenomenon - the incredible memorability of microblogs - and explored several potential mechanisms for this effect. The results from the first experiment suggested that memory for Facebook posts is remarkably strong, significantly stronger than that observed either for sentences drawn from published books or for faces. The second experiment showed that this effect was not due to the Facebook posts naturally producing deep social encoding; when people were asked to do just such deep social encoding, it enhanced memorability just as much for the Facebook posts as for the book sentences. If the advantage of the posts were due to people, or at least some reasonable fraction of them, already doing such social encoding, telling them to do so would have had a relatively small effect, not the large effect that was demonstrated. In the final experiment, we tested three factors that could contribute to the superiority of the posts and found some evidence for each. Text that is designed to be complete is indeed remembered better, as was shown by the superiority, in memorability, of headlines over sentences drawn from the bodies of CNN articles. More gossipy text also seems
Table 1 Average proportions (with standard deviations) of low-frequency words per text type in Experiment 3

\begin{tabular}{lccc}
\hline Text Type & Breaking News & Entertainment News & Both News Types \\
\hline Comments & $.16(.13)$ & $.21(.15)$ & $.18(.14)$ \\
Headlines & $.34(.18)$ & $.40(.19)$ & $.37(.19)$ \\
Sentences & $.19(.12)$ & $.21(.13)$ & $.20(.13)$ \\
All text types & $.23(.16)$ & $.27(.18)$ & $.25(.18)$ \\
\hline
\end{tabular}


advantaged, with text drawn from entertainment news beating text drawn from breaking news. Finally, it also seems that sentences written casually by lay people, without professional, or perhaps any, editing, are especially readily remembered, as evidenced by the memorability of the comments posted at the ends of entertainment and news articles.

Related to this idea that casually generated language may be remembered better, Keenan, MacWhinney, and Mayhew (1977) examined memory for conversational sentences uttered by people who participants either did or did not know, and that had high or low interactive content (i.e., sentences whose meaning relied, or did not rely, on knowledge of the specific speaker's intentions with regard to the particular listener), using taped lunchroom conversations. The authors concluded that conversational sentences were remembered better when people knew the speaker and when the sentences were high in interactional content. The memory strength of the Facebook posts and article comments is not exactly parallel, as participants were unlikely to know any of the authors, and so could not tie the utterances to other details of their originators. However, both the posts and comments - and, to some extent, the entertainment stories and headlines - do have a conversational, spontaneous tone (microblogging is, after all, part way to virtual chatting), and thus may not be entirely unrelated to hearing personal details from people whom one knows. Further research would be needed to determine whether this phenomenon applies to all social media (e.g., tweets or text messages), and even whether it applies to notes that one writes oneself, such as diary entries.

Many may consider the Facebook postings and article comments to be vacuous, narcissistic, or vapid, but they are thoughts that their writers considered worth sharing. A philosophical treatise by Immanuel Kant may be more profound, and more edifying to remember, than the average Facebook post or article comment, but his writings may not be tuned so precisely to what our minds effortlessly encode. These especially memorable Facebook posts and reader comments, generated by ordinary people, may be far closer than professionally crafted sentences to tapping into the basic language capacities of our minds. Perhaps the very sentences that are so effortlessly generated are, for that reason, the same ones that are readily remembered. Some sentences - and, most likely, those without careful editing, polishing, and perfecting - are naturally more "mind-ready." The advantage of entertainment over news, as well as the advantages of Facebook posts over book sentences and of comments over the headlines and text of articles, may reflect such a status. Perhaps these effortlessly occurring proclamations help foster social belongingness that may extend to online communities. As a result, this type of shared information does have a privileged status and is remembered more readily (Nairne \& Pandeirada, 2010).
It seems that, with the growth of blogging, text messaging, and the like, written language has moved closer to natural speech, with less editing and contemplation than was needed not only when the writing was done by monks with goose-feather quills or by Gutenberg with moveable type, but even when it is done by authors sitting patiently at their own keyboards. In Japan, several top-selling novels were written in the form of a series of cellphone text messages (www.nytimes.com/2008/01/20/world/asia/ 20japan.html). In television, likewise, "reality" programming has been on the increase, moving away from careful scripting and plotting to something close to what humans naturally put out, and so perhaps just as naturally take in.

In education, spoken lectures have long been viewed as a useful adjunct to written texts, even when the latter have been more polished, thoughtful, and complete. Consistent with our findings, the nature of the communication may make the information more memorable, with the professor generating natural speech. Perhaps, though, textbooks written as tweets would render the faculty obsolete.

Author note We thank J. T. Wixted for his comments on this work, as well as Travis Seale-Carlisle, Kim Huynh, Johanna McElfresh, Michelle Niku, Katelyn Steele, Lisa Teachanarong, Brenda Wu, and Allison Yee for data collection.

\section{Appendix: Sample stimuli}

\section{Facebook Posts}

i am 7,689 days old...

The library is a place to study, not to talk on your phone My math professor told me that I was one of his brightest students

Love clean sheets :)

$\mathrm{Bc}$ sometimes it makes me wonder

\section{Sentences From Books}

How did he end up in this family?

Underneath the mass of facial hair beamed a large smile.

Even honor had its limits.

Cody raised his .40 Sig Sauer in a shooter's grip.

My throat was burning from screaming so loudly.

\section{CNN Breaking News Headlines}

Sixth person dies after stage collapse at Indiana State Fair EU panel calls for embargo on Syrian oil as reports of deaths mount

Justice Department charges 91 in \$295 million Medicare fraud scheme 


\section{CNN Breaking News Sentences}

Americans respond to decisiveness.

At least 29 deaths were reported from a defiant outpouring of mass demonstrations Friday.

He was arrested Thursday and was taken before federal investigators for interrogation.

\section{CNN Breaking News Comments}

I am an unemployed teacher in the deep south.

Attacking schoolchildren and funerals. Yep, that's the brave Taliban, alright.

I told you so, but never listen going again to the wrong direction.

\section{CNN Entertainment Headlines}

Housewives' star Michaele Salahi assures deputy she's not kidnapped

Netflix now enforcing streaming limit

Phish to host benefit for Vermont flood victims

\section{CNN Entertainment Sentences}

Ryan O'Neal attended the hearing Wednesday, but did not address the court.

The actor attributes his burst of heroism to feeling limber. We believe at one point, he even breaks out the running man.

\section{CNN Entertainment Comments}

Is this lady really a model? Wow she looks awful!

No talent hack, I should feed him to the lizards.

We will never forget that day and how it changed our lives.

\section{References}

Baumeister, R. F., \& Leary, M. R. (1995). The need to belong: Desire for interpersonal attachments as a fundamental human motivation. Psychological Bulletin, 117, 497-529. doi:10.1037/00332909.117.3.497

Belmore, S. M. (1982). The role of imagery in recognition memory for sentences. Acta Psychologica, 50, 107-115.

Craik, F. I. M., \& Lockhart, R. S. (1972). Levels of processing: A framework for memory research. Journal of Verbal Learning and Verbal Behavior, 11, 671-684. doi:10.1016/S0022-5371 (72)80001-X
Dunbar, R. I. M. (2004). Gossip in evolutionary perspective. Review of General Psychology, 8, 100-110.

Emery, N. J. (2000). The eyes have it: The neuroethology, function and evolution of social gaze. Neuroscience and Biobehavioral Reviews, 24, 581-604.

Facebook.com. (2011). Facebook statistics [Website information]. Retrieved June 20, 2011, from www.facebook.com/press/info.php? statistics

Glanzer, M., \& Bowles, N. (1976). Analysis of the word-frequency effect in recognition memory. Journal of Experimental Psychology: Human Learning and Memory, 2, 21-31.

Griggs, B. (2009, August 20). The 12 most annoying types of Facebookers [Online article]. Retrieved from http://articles.cnn.com/ 2009-08-20/tech/annoying.facebook.updaters_1_facebook-usersfriend-online-social-networks? s=PM:TECH

Kang, S. H. K., McDermott, K. B., \& Cohen, S. M. (2008). The mnemonic advantage of processing fitness-relevant information. Memory \& Cognition, 36, 1151-1156. doi:10.3758/MC.36.6.1151

Kanwisher, N., McDermott, J., \& Chun, M. M. (1997). The fusiform face area: A module in human extrastriate cortex specialized for face perception. Journal of Neuroscience, 17, 4302-4311.

Keenan, J. M., MacWhinney, B., \& Mayhew, D. (1977). Pragmatics in memory: A study of natural conversation. Journal of Verbal Learning and Verbal Behavior, 16, 549-560. doi:10.1016/ S0022-5371(77)80018-2

Klein, S. B., Cosmides, L., Gangi, C. E., Jackson, B., \& Tooby, J. (2009). Evolution and episodic memory: An analysis and demonstration of a social function of episodic recollection. Social Cognition, 27, 283-319.

Macmillan, N. A., \& Creelman, C. D. (2005). Detection theory: A user's guide (2nd ed.). Mahwah, NJ: Erlbaum.

Mandler, G. (1979). Organization and repetition: Organizational principles with special reference to rote learning. In L.-G. Nilsson (Ed.), Perspectives on memory research (pp. 293-327). Hillsdale, NJ: Erlbaum.

Manns, J. R., Hopkins, R. O., Reed, J. M., Kitchener, E. G., \& Squire, L. R. (2003). Recognition memory and the human hippocampus. Neuron, 37, 171-180.

Merz, C. J., Wolf, C. T., \& Hennig, J. (2010). Stress impairs retrieval of socially relevant information. Behavioral Neuroscience, 124, 288-293.

Mickes, L., Wixted, J. T., \& Wais, P. E. (2007). A direct test of the unequal-variance signal detection model of recognition memory. Psychonomic Bulletin \& Review, 14, 858-865. doi:10.3758/ BF03194112

Nairne, J. S., \& Pandeirada, J. N. S. (2010). Adaptive memory: Nature's criterion and the functionalist agenda. The American Journal of Psychology, 123, 381-390.

Nairne, J. S., Pandeirada, J. N. S., \& Thompson, S. R. (2008). Adaptive memory: The comparative value of survival processing. Psychological Science, 19, 176-180. doi:10.1111/j.1467-9280.2008.02064.x

Nairne, J. S., Thompson, S. R., \& Pandeirada, J. N. S. (2007). Adaptive memory: Survival processing enhances retention. Journal of Experimental Psychology: Learning, Memory, and Cognition, 33, 263-273. doi:10.1037/0278-7393.33.2.263

Schiefele, U., \& Krapp, A. (1996). Topic interest and free recall of expository text. Learning and Individual Differences, 8, 141-160.

Takahashi, T., Ikeda, K., Ishikawa, M., Tsukasaki, T., Nakama, D., Tanida, S., \& Kameda, T. (2004). Social stress-induced cortisol elevation acutely impairs social memory in humans. Neuroscience Letters, 363, 125-130. 